\title{
E09 A GEOSTATISTICAL APPROACH TO HISTORY MATCHING PRESSURE AND FLOW DATA ON NON-UNIFORM GRIDS
}

Omer Inanc TUREYEN and Jef CAERS

Stanford University, Petroleum Engineering, Stanford, CA94305-2220, USA

\begin{abstract}
Fine scale heterogeneities can have significant effects on flow performances in subsurface formations. Fine-scale geostatistical realizations generated from well-log and seismic data are required to quantify the impact of such heterogeneities on flow. These realizations in many cases require number of grid cells on the order of $10^{6}-10^{7}$, hence making it too demanding CPU wise to directly history match geostatistical models. Non-uniformly gridding these models for emphasizing the heterogeneities does not necessarily present a solution since the practice of geostatistics on non-uniform grids is inefficient.

In this paper we provide an entirely new approach to these problems by proposing a solution which is geostatistics based yet having the flexibility to work on non-uniformly gridded models. Hence coupling geostatistics with non-uniformly upscaled models results in an effective history matching technique incorporating all information at their scales. With the proposed approach we mainly target three advantages. Well and seismic data will still be effectively incorporated in the realizations. Flow simulations will be performed on non-uniformly upscaled models hence will be relatively fast. Finally the resulting field which is history matched will include the geology and will be on a non-uniformly gridded model for emphasizing heterogeneities or desired specifications, hence future predictions can be made fast, no posterior upscaling is required.
\end{abstract}

\section{Introduction}

History matching is an important step in reservoir characterization; conditioning realizations to well-log or seismic data alone is not enough. One major challenge of data integration lies in the fact that each data has its own resolution and area of coverage. Well-log data has high resolution but are sparse. Production data informs a much larger scale but carries little information on local heterogeneity. Since well-log data are considered to be hard data, fine scale gridding is needed to include this type of data in the final reservoir model. History matching techniques, which typically require a large number of flow simulations, cannot be directly applied to the high resolution geostatistical models because of the CPU costs of current finite difference simulators.

History matching methods such as the Sequential Self Calibration Method (Wen, Deutsch and Cullik, 1998) address this problem by upscaling the initial fine scale geostatistical model. First, an initial seed realization with up to $10^{6}-10^{7}$ grid cells is generated. Next, this initial model is upscaled uniformly to $10^{3}-10^{4}$ grid cells rendering flow simulations feasible. The initial upscaled model is perturbed until history match is achieved. Next, the coarse scale history-matched model is downscaled using block conditioning algorithms (Tran et al., 1999) for the following reasons:

$8^{\text {th }}$ European Conference on the Mathematics of Oil Recovery - Freiberg, Germany, 3 - 6 September 2002 
- The coarse-scale history matched models do not necessarily honor the fine scale well-log data.

- Fine-scale realizations are often requested as input to more sophisticated non-uniform upscaling methods, and to local grid refinement.

However this last downscaling step presents various problems such as:

- The fine scale model may not match anymore tightly the production data since the downscaling procedure adds additional short scale structures to the model. Also the process of downscaling does not provide a single unique answer as upscaling does, Ying (2001).

- The downscaled realizations need to be upscaled again to make flow simulations feasible. There is again no guarantee that this newly upscaled model matches history.

In summary, many history matching methods tend to go through a sequence of upscalings and downscaling. This might not be an efficient way to integrate data of variable scales since the sequence of upscaling and downscaling affects the matching of information at different scales.

One alternative to this problem is to generate directly non-uniformly gridded models. However this would call for generating spatially correlated block effective or average properties defined on different support volumes. Geostatistics is ill-equipped to deal with such variables.

We propose to construct in parallel a fine scale and the corresponding non-uniform coarse model. We make the upscaling phase part of the history matching process instead of a prior or posterior process. Perturbations to the permeability field (or any other property that effects the production signal) are performed on the fine scale model, that fine scale model is then upscaled after each perturbation; in particular flow simulation is done (rapidly) on the coarse model. The fine scale model perturbations are guided by the results of the coarse model simulation results. This approach could provide three advantages:

1. We obtain two end-results: a fine scale model, and a corresponding coarse, non-uniformly gridded, model that is history matched. The latter can be used directly as input to the reservoir simulator.

2. The coarse non-uniform model includes the relevant high and low permeability information from wells and seismic as these condition the parallel fine scale model.

3. All history match flow simulations are done on the coarse, allowing a speed-up of the history match effort.

\section{History Matching}

Before the essence of our proposed method we review shortly the history matching algorithm used. Although it should be stressed that any other algorithm can be used for this purpose.

In this paper we use the method of history matching by the gradual deformation first presented in Roggero and Hu (1997), later modified by Ying and Hernandez (2000) to account for well-log data. Many types of algorithms exist which are shortly reviewed. The original gradual deformation method combines Gaussian random functions while maintaining their statistical properties (histogram and variogram). In a first step, one takes three individual fine scale realizations which honor the hard data and apply a normal score transform to each of them. Next, the normal score realizations, are pixel-wise linearly combined through a single parameter $r$ in a way so that the resulting normal score realization honors all hard data and the statistics of the initial realizations. The resulting realization is then back transformed into the data space. For any given parameter value $r$ the resulting realization is evaluated in a flow simulator resulting in the 
desired objective function. It is now a matter of finding the optimal value $r^{\text {opt }}$ leading to a minimized objective function. Since only one parameter $(r)$ needs to be optimized we can apply any existing one dimensional optimization code, e.g. Dekker Brent [6]. This process is repeated until a history match is achieved.

Another type of gradual deformation relies on perturbation of random numbers. In this approach, deformations are achieved by gradually modifying the random numbers used in generating stochastic realizations using the sequential simulation methodology. This perturbation of random numbers is parameterized by a single parameter $r$ that can be optimized, as discussed above. In this approach, any type of sequential simulation method, such as facies-based categorical methods, can be used, not necessarily being restricted by Gaussian assumptions.

\section{Proposed Method}

The deformation algorithms explained in the previous section require multiple flow simulations in the inner loop to check matching history. Such flow simulations are CPU demanding if performed on high resolution fine scale realizations. To speed up the process we propose to couple the deformation algorithm with upscaling techniques. Fine scale realizations are upscaled non-uniformly, then flow simulation is performed on the upscaled model, and the difference between calculated and measured production data is based on that upscaled model.

The fine scale geostatistics and the corresponding coarse scale flow simulation grids although parallel are kept separated. Multiple but fast flow simulations are performed on the coarse scale grid while data conditioning and statistical continuity reproduction is obtained from the fine scale grid. Non-uniform upscaling on the other hand will preserve important regions that would have great impact on flow.

The remaining task is to provide an efficient non-uniform upgridding and related upscaling method. The upscaling needs to be CPU efficient.

\section{Upgridding}

Many grid cell upscaling methods exist, either static or flow based. In this study we have chosen to use the flow based upgridding technique proposed by Durlofsky et al. (1995). This technique targets the high permeability and high flow regions. The technique requires a single phase, incompressible flow simulation to be run on the fine scale geostatistical model. A velocity field $u_{x, y}$ (for 2-D reservoirs, with $\mathrm{y}$ the vertical direction) is derived which a non-uniform grid is determined. Once the velocity field $u_{i, j}$ ( $\mathrm{i}, \mathrm{j}$ are the integer indices for the fine scale grid cell) is determined they are averaged along each row $j$ as:

$$
u_{j}^{a v g}=\frac{\sum_{i=1}^{n_{x}} u_{i, j}}{n_{x}}
$$

where $n_{x}$ represents the number of grid cells in the $\mathrm{x}$ direction. Similarly the average velocity for each column $i$ is calculated as:

$$
u_{i}^{a v g}=\frac{\sum_{j=1}^{n_{y}} u_{i, j}}{n_{y}}
$$

where $n_{y}$ represents the number of grid cells in the y direction. Next, the row average velocities are standardized as: 


$$
u_{j}^{s t}=\frac{u_{j}^{a v g}}{\max _{j}\left(u_{j}^{a v g}, j=1, \ldots, n_{y}\right)}
$$

Similarly for the columns:

$$
u_{i}^{s t}=\frac{u_{i}^{\text {avg }}}{\max _{i}\left(u_{i}^{\text {avg }}, i=1, \ldots, n_{x}\right)}
$$

We present first the algorithm for upgridding in only one dimension (uplayering) then proceed to the general 3-D case.

In order to preserve important high permeability layers, we preserve layers with high standardized velocity and upgrid layers with low $u^{s t}$. The desired level of upgridding determines the threshold velocity above which high velocity layers should be preserved. For example for a very coarse upgridding requirement, one would expect high velocity layers to be combined. To achieve an upgridding method that a certain target coarse grid dimension, we approximately introduce the following tuning parameters:

$u^{\max }$ is the maximum acceptable cumulative velocity of all fine-scale layers composing any coarse-scale upgridded layer. If $u^{\max }$ is set to zero then the corresponding upgridded model will have the same number of layers as that of the fine scale model. As $u^{\max }$ is increased the dimensions of the coarse model are decreased until $u^{\max }$ reaches the sum of all layers. At this point the coarse model would only be composed of a single layer.

$n^{\max }$ is the maximum allowable number of fine-scale layers composing any coarse-scale upgridded layer. If $n^{\max }$ is set to 1 then the corresponding upgridded model will have the same number of layers as that of the fine scale model. As $n^{\max }$ is increased then $u^{\max }$ will become more dominant in determining the gridding.

\section{Case Studies}

In this section demonstarte the approach in 3 case studies. The first two will be simple 2D cases which we hope will give a clear understanding to the proposed method. Finally the third case will be a $3 \mathrm{D}$ quarter five spot pattern.

\section{A Gaussian Model}

The first case study is done on a 2D gaussian model. In the first model we focus on layering. The reference permeability field and its corresponding fractional flow curve is given in Figure-1. The production history lasts for 500 days under fixed bottom hole pressures of 5500psi and 4500 psi. Figure-2a represents the behavior of the objective function for two different starting points. Both objective functions tend to reach a plateau around 12 iterations, indicating that the deformation parameter $r$ has been optimized 12 times. Figure-2b shows the progress of fractional flow curves during iterations. At the end of iteration 20 we have a reasonable history match. Figure- 3 shows the progression of the permeability fields along with the reference permeability field and its corresponding non-uniformly upscaled version. As we progress in matching the history, we keep the fine scale and the coarse scale models in parallel. All of the fine scale models honor the required spatial statistics, ultimately two end models are obtained; a fine scale model and a coarse scale model which we can use to make fast future predictions. Figure-4a illustrates the flow responses of realizations conditional only to the hard data and the variogram. The scatter is relatively large, especially in terms of break-through times see Figure-4a. Note that all flow 
responses are evaluated on the fine grid. Figure-4b. shows results after history matching production data limited to the first 500 days. A good bracketing of future predictions up to 2000 days is achieved. Note that flow responses are now calculated on the coarse grid.

\section{A Facies Model}

In this section we present the results of the proposed method applied on permeability distributions with discrete facies. We use the SNESIM (Strebelle, 2002) code to generate facies using the training image depicted in Figure-5a. Each of the four facies is then assigned a constant permeability of $3,30,300$ and 3000 md respectively. As gradual deformation method we apply the gradual deformation of random numbers methodology.

The injection/production scheme is taken the same as the first case in the previous section. The reference field and the corresponding fractional flow curves are given in Figure $5 \mathrm{~b}$ and $5 \mathrm{c}$ respectively. As shown from Figure-5b layering is emphasized by assigning the lowest and highest permeabilities to the facies that extend horizontally. Figure-6a shows the fractional flow of 20 unconditional realizations built from the same training image of Figure 5a resulting in a wide scatter. History matching decreases the uncertainty of the future predictions as shown in Figure-6b.

\section{Conclusions}

The following conclusions are obtained from this study:

1. With the new proposed method the CPU demand of flow simulators is significantly decreased, since we are running flow simulations on coarse models, hence the entire history matching process becomes relatively fast.

2. Geostatistics on the uniform fine scale has successfully been coupled with non-uniform coarse models simply by making the upscaling process a part of the history matching, hence making the algorithm effective in terms of integrating various kinds of data at their relevant scales.

3. The resulting history matched realization, is upscaled and non-uniformly gridded. Future predictions in this case can be made faster. Also no upscaling errors are introduced. These coarse models still contain important information about the field's obtained from the finescale model.

\section{References}

[1] Wen, X.H., Deutsch, C and Cullik, A.S., Integrating pressure and fractional flow data in reservoir modelling with fast streamline based inverse methods. Paper SPE 48971, prepared for presentation at the 1998 SPE/ATCE, New Orleans, 27-30 Sept 1998

[2] Ying, Z., 2001. Uncertainty of Flow Responses After Conditioning to Block Averages, Stanford Center for Reservoir Forecasting, Annual Report No 14, Stanford, USA.

[3] Roggero, F. and Hu, L.Y., 1998 Gradual deformation of continuous geostatistical models for history matching. Paper SPE 49004 prepared for the SPE ATCE, New Orleans, September 1998.

[4] Ying, Z., Gomez, H., 2000. An Improved Deformation Algorithm for Automatic History Matching, presented at the $13^{\text {th }}$ SCRF Affiliates meeting, Stanford

[5] Press, W.H., Teukolsky, S.A., Vetterling, W.T., Flannery, B.P., 1989. Numerical Recipes in Fortran, Second Edition, pp. 397

$8^{\text {th }}$ European Conference on the Mathematics of Oil Recovery - Freiberg, Germany, 3 - 6 September 2002 
[6] Caers, J., 2002. Methods for History Matching Under Geological Constraints, In Proceeding to ECMOR VIII, $8^{\text {th }}$ European Conference on Mathematics of Oil Recovery, 2002

[7] Strebelle, S., 2001. Conditional Simulation of Complex Geological Structures Using Multiple point Statistics, Mathematical Geology 34(1) 1-26.

[8] Durlofsky, L.J., Jones, R.C., Milliken, W.J., 1995. A Non-Uniform Coarsening Approach for the Scale-Up of Displacement Processes in Heterogeneous Porous Media, Advances in Water Resources, Vol. 20, pp. 335-347 


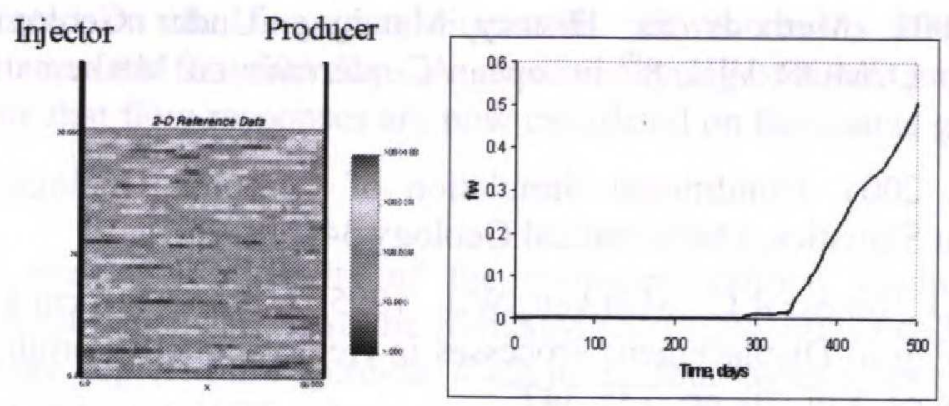

Figure-1: The reference permeability field and its corresponding fractional flow curve for the gaussian layered case.
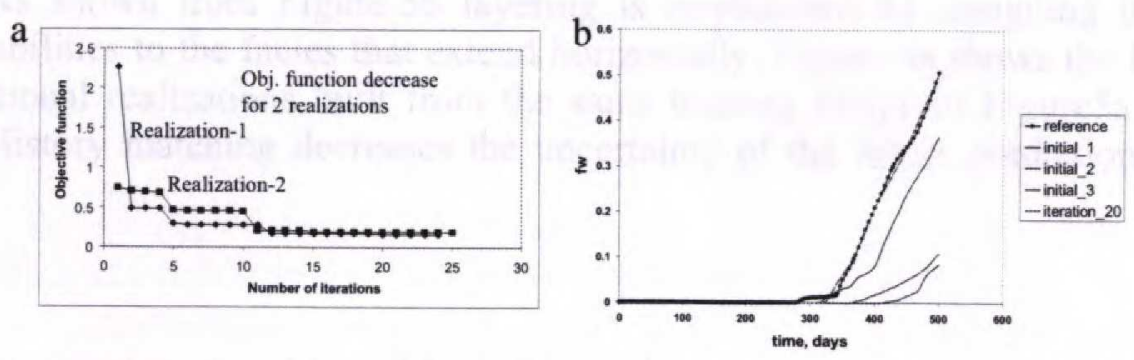

Figure-2: (a) Progression of the objective function, (b) Progression of the fractional flow curves.

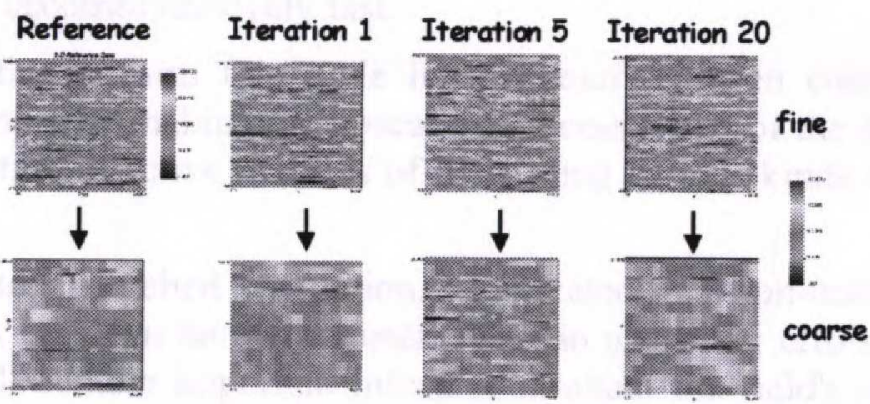

Figure-3: Progression of the permeability field during history matching.
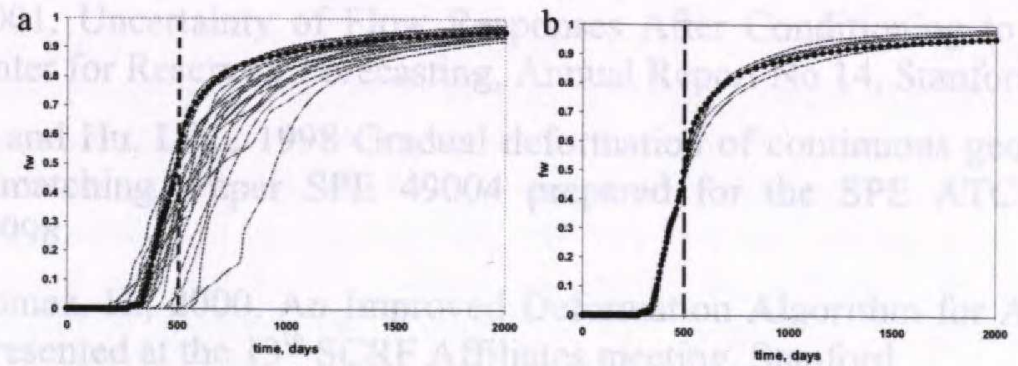

Figure-4: (a) Flow responses before history matching, (b) Flow responses after history matching. 
a

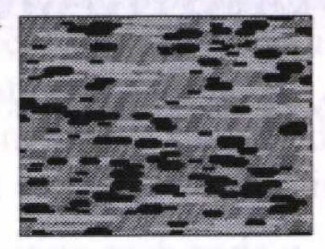

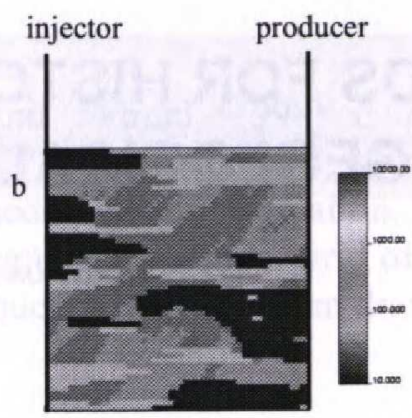

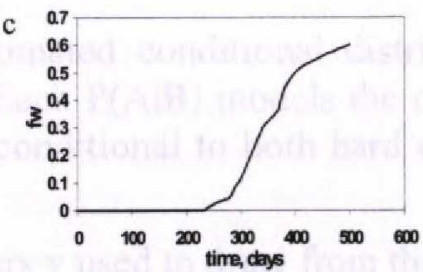

Figure-5: (a) The training image (400x150) used to create the reference permeability field for the facies model, (b) The refernce permeability field $(50 \times 50)$, (c) Flow response of the reference permeability field.
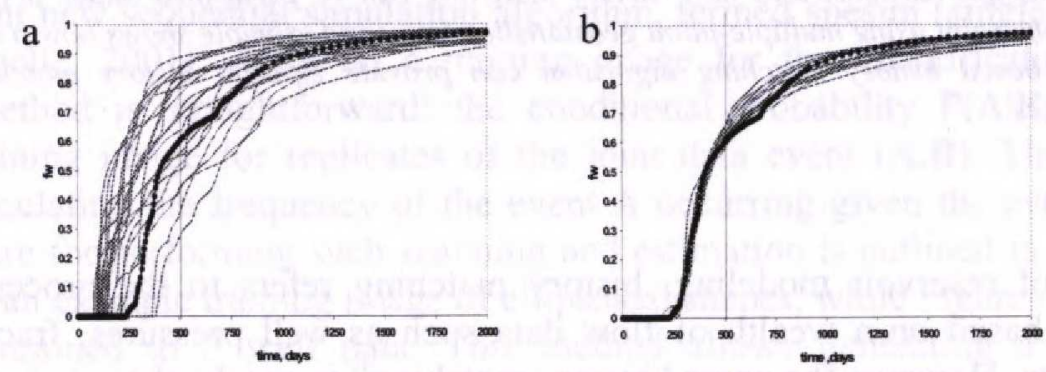

Figure-4: (a) Flow responses before history matching, (b) Flow responses after history matching. 